\title{
Efficient Computation of Spinning Modal Radiation Through an Engine Bypass Duct
}

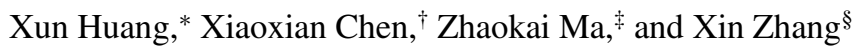 \\ University of Southampton, Southampton, England SO17 1BJ, United Kingdom
}

DOI: $\underline{10.2514 / 1.31136}$

\begin{abstract}
The aim of this work is to accurately and efficiently predict sound radiation out of a duct with flow. The sound propagation inside a generic engine bypass duct, refractions by the shear layer of the exhaust flow, and propagation in the near field are the main focus of the study. The prediction uses either a modified form of linearized Euler equations or an alternative model based on acoustic perturbation equations, which were extended to cylindrical coordinates. The two models were compared on a canonical case of sound propagation out of a semi-infinite duct with flow. Good agreements between the predictions were achieved. The more general case of a generic aircraft engine bypass duct with flow was then investigated with the technique of adaptive mesh refinement to increase the computational efficiency. The results show that both linearized Euler equations and acoustic perturbation equations models can predict the near-field sound propagation and far-field directivity. The acoustic perturbation equations model, however, is more adaptive for its suitability to an arbitrary background mean flow.
\end{abstract}

\section{Nomenclature}

$\begin{array}{ll}\mathbf{A} & =\text { operator matrix for acoustic perturbation equations } \\ C_{0} & =\text { sound speed } \\ f, \tilde{\mathbf{F}} & =\text { Fourier-Laplace transform pair } \\ H & =\text { Heaviside function } \\ J, Y & =\text { first and second kind of Bessel functions } \\ k & =\text { blade passing angular frequency } \\ k_{a}, k_{r} & =\text { axial and radial wave numbers } \\ L & =\text { length } \\ M_{0}, M_{j} & =\text { Mach number outside and inside jet } \\ m, n & =\text { circumferential and radial modes } \\ p & =\text { pressure } \\ S_{1-4} & =\text { source terms for linearized Euler equations } \\ s & =\text { complex parameter for Laplace transform } \\ \mathbf{T}^{a} & =\text { acoustic filter } \\ t & =\text { sound wave propagation time } \\ u, v, w & =\text { velocity in cylindrical coordinates } \\ w_{t}^{\prime} & =\partial w^{\prime} / \partial t \\ x, y, z & =\text { Cartesian coordinates } \\ x, r, \theta & =\text { cylindrical coordinates } \\ x_{1-4} & =\text { eigenvectors of } \mathbf{A} \\ \alpha, \beta & =\text { wave number in the } x \text { and } r \text { coordinates } \\ \lambda_{1-4} & =\text { eigenvalues of } \mathbf{A} \\ \rho & =\text { density } \\ \phi & =\text { far-field observer angle }\end{array}$

Subscripts

$m, n=$ circumferential mode and radial mode $0=$ background flow value

Received 19 March 2007; revision received 2 January 2008; accepted for publication 14 February 2008. Copyright @ 2008 by Xun Huang and Xin Zhang. Published by the American Institute of Aeronautics and Astronautics, Inc., with permission. Copies of this paper may be made for personal or internal use, on condition that the copier pay the $\$ 10.00$ percopy fee to the Copyright Clearance Center, Inc., 222 Rosewood Drive, Danvers, MA 01923; include the code 0001-1452/08 $\$ 10.00$ in correspondence with the CCC.

*Lecturer, Aeronautics and Astronautics, School of Engineering Sciences; xunger@ soton.ac.uk. AIAA Member.

'Research Fellow, Aeronautics and Astronautics, School of Engineering Sciences; x.chen@soton.ac.uk.

*Research Fellow, Aeronautics and Astronautics, School of Engineering Sciences; mazhk@soton.ac.uk.

${ }^{\S}$ Professor, Aeronautics and Astronautics, School of Engineering Sciences; x.zhang1@soton.ac.uk. Associated Fellow AIAA.

\begin{tabular}{|c|c|c|}
\hline \multicolumn{3}{|c|}{ Superscripts } \\
\hline$a$ & $=$ & acoustic mode part \\
\hline$T$ & $=$ & transpose of a matrix \\
\hline ' & $=$ & acoustic disturbance value \\
\hline & $=$ & Fourier-Laplace transformed variable \\
\hline$*$ & $=$ & reference value \\
\hline
\end{tabular}

\section{Introduction}

$\mathbf{T}$ HE development of high bypass ratio turbofan engines has led to more prominent tonal noise, which is generated by the fan assembly. An accurate model of propagation of tonal noise within and away from the engine duct would provide a valuable tool in determining suitable methods to alleviate the fan tonal noise and assess environmental impact. In the case of radiation from either a bypass duct or a core exhaust nozzle, as shown in Fig. 1, there are issues associated with the presence of a shear layer between exhaust mean flow and external mean flow. Refractive effects due to the presence of the shear flow can change noise radiation patterns. The physical process of noise generation and radiation is governed by the Navier-Stokes equations. At present, a full numerical solution of noise generation, propagation, and radiation process using the Navier-Stokes equations is quite expensive. However, certain aspects of the noise propagation and radiation process can be modeled by linearized equations. For example, in the duct downstream of the rotor-stator region of an aircraft engine, where nonlinear and thermal noise generation effects are minimal, the propagation of the rotor-stator noise can be studied using the inviscid linearized equations about the background flow. A significant amount of research has been undertaken to develop analytical and computational methods for bypass duct noise problems. An extensive review of the existing analytical methods was presented by Gabard and Astley [1]. Of the main numerical approaches in computational aeroacoustics (CAA), boundary element methods [2] are confined to problems of acoustics through uniform background flows. Finite/infinite element methods [ $[3, \underline{4}]$ are generally restricted to acoustic propagation through irrotational steady flows, and the high-order finite difference methods $[5,6]$ on structured grids with Euler or linearized Euler equations $(\bar{L} \bar{E} \bar{E})$ are much too general in terms of governing physics [7] and parallel computing portability. A different spatial discretization method for LEE, using the discontinuous Galerkin method on unstructured grids, was reported in the literature [8] recently to solve bypass duct problems. 


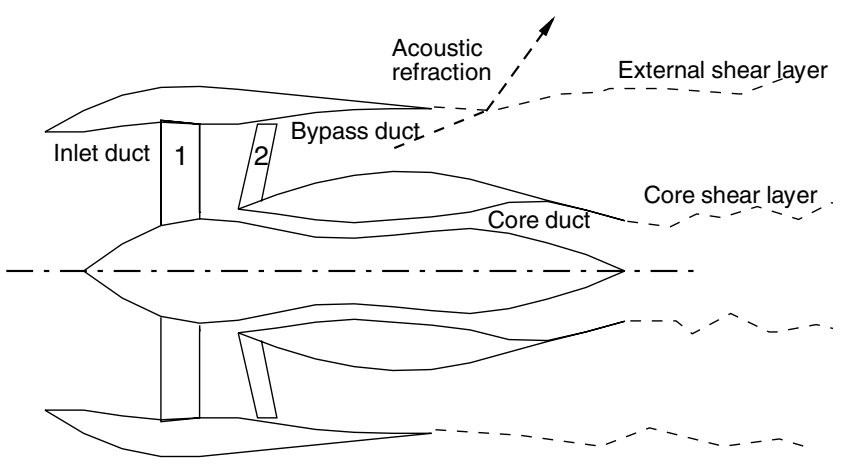

Fig. 1 Tonal noise radiation from bypass duct; 1: rotor, 2: stator.

To accurately and efficiently predict sound radiation out of a duct with flow, a CAA method based on the LEE model was presented in our previous work [7,9-12]. A 2.5-D LEE model was developed to solve sound radiation out of an axisymmetric duct on a 2-D computational domain $[\underline{9}, 10]$. The incoming spinning modes were realized through an absorbing nonreflecting boundary treatment, which admits incoming waves and damps spurious waves generated by the numerical methods [11]. A time-domain impedance boundary condition was implemented for studying the attenuation effectiveness of liners that are installed on the wall of an engine bypass duct [7]. The technique of parallel adaptive mesh refinement (AMR) was also developed and used to reduce the computational time for an engine intake case by increasing grid resolution only in the desired area [12]. Up to now, the duct geometry was assumed axisymmetric, and the background flow axisymmetric with no swirl component.

The LEE model, however, describes not only the acoustic mode but also vorticity modes that are unstable in a shear flow. The unstable components develop exponentially to finally corrupt the desired acoustic solutions. It was a common practice to avoid the numerical instabilities by solving the problem in the frequency domain $[3,13,14]$. To use the current time-domain code, the LEE model was "degraded" by removing several terms that are singular in a sheared background flow [7]. The modified equations were termed revised 2.5-D LEE, which were validated against Munt's asymptotic solution [15] in the canonical case of sound radiation out of a semiinfinite duct with flow [10]. A similar modification for LEE can be found in the literature [1] $]$. To soothe the concerns [13] raised over the nonphysical modification, an alternative model based on acoustic perturbation equations (APE) $[\underline{17}, \underline{18}]$ was employed in this work. The original APE model was modified for axisymmetric applications. For convenience, the modified version is still called APE in this paper.

The homogeneous APE without any sound source are actually perturbation equations introduced by Pierce [19]. The wave equation for sound propagation in an inhomogeneous fluid was approximated with ambient properties and flow that vary with position and time. Assuming the characteristic length and time scales for the acoustic disturbances were smaller than the corresponding scales for the background flow, a concise wave equation was developed by only taking into account derivatives up to the first order of the inhomogeneities and the ambient unsteadiness. As a special feature, nonacoustic modes, otherwise present in the LEE model, can be removed in the APE model. However, in general inhomogeneous flows, an interaction between acoustic and nonacoustic modes takes place which is not included in the APE model. The diffraction of acoustic waves at sharp edges would also generate vorticity waves due to a Kutta condition, but these vorticity waves are also missing in the APE simulation. The APE model, however, was used in this work only to approximate refraction effects of spinning modal sound waves. It was assumed that, for diffraction problems, imposing the Kutta condition or not has almost no impact on the acoustic field.
The technique of AMR was employed to efficiently compute the realistic case of sound radiation out of a generic engine duct with flow on an adaptively refined mesh. Generally, AMR is efficient and effective in treating problems with multiple spatial and temporal scales [20]. The technique represents the computational domain as hierarchal refinement levels and increases the grid resolution only in areas of interest. The computational efficiency is improved by reducing the required number of computational cells. The existing AMR applications [21,22] generally employ a blockstructured AMR algorithm. It involves 1) representing the 2-D/3-D hierarchical computational domain in blocks; 2) connecting the generated blocks in a quadtree/octree data structure; 3) estimating local truncation errors at all grid points and identifying blocks with excessive errors; 4) regridding the identified blocks by superimposing or removing blocks to accommodate changes in flow physics; and 5) redistributing the computational load between processors to maintain dynamic load balancing. This procedure is operated recursively until either a given refinement/coarsening level is reached or a predefined local truncation error level has been met.

Both the revised 2.5-D LEE model and the APE model were used in this work. Along with numerical implementations, both models are introduced in Sec. II. To compare the two models, the canonical case of sound radiation out of a semi-infinite duct with flow is investigated in Sec. III. A more generic case of sound radiation out of an aircraft engine bypass duct is studied with the AMR technique in Sec. IV, where the AMR implementation, operation procedure, and the computational efficiency are briefly introduced. Section $\underline{\mathrm{V}}$ summarizes the two models and results.

\section{Numerical Models}

\section{A. Propagation: Linearized Euler Equations}

This research focused on the prediction of propagation and radiation of spinning modes generated by the engine fan and fan/ stator flow interactions. As the acoustic perturbation is small compared with the background flow, sound wave propagation can be modeled by LEE. For typical axisymmetric engine geometry, it is more convenient to formulate LEE in the cylindrical coordinate. Solutions of the full 3-D equations, however, are expensive. Assuming that the acoustic disturbances are restricted to the blade passing frequency and its harmonics, it is possible to write the disturbances at each frequency by a Fourier series in terms of circumferential modes. For instance, a series representing the pressure disturbance $p^{\prime}$ at a single angular frequency $k$ is

$$
p^{\prime}=\sum_{m=-\infty}^{\infty} p_{m}^{\prime}(x, r) e^{[i(k t-m \theta)]}
$$

where $x$ is the axial coordinate, $r$ is the radial coordinate, $i$ is the imaginary unit, $t$ is the sound wave propagation time, $m$ is the circumferential mode, and $\theta$ is the circumferential angle. Subsequently, there are two important relations for the circumferential velocity disturbance $w^{\prime}$ and the pressure disturbance $p^{\prime}$. They are

$$
\frac{\partial w^{\prime}}{\partial \theta}=-\frac{m}{k} \frac{\partial w^{\prime}}{\partial t}, \quad \frac{\partial^{2} p^{\prime}}{\partial t \partial \theta}=m k p^{\prime}
$$

By using Eq. (2), the general 3-D LEE in cylindrical coordinates could be simplified to a set of 2-D equations, which are generally termed 2.5-D LEE equations [9]. The complete governing equations for a single frequency $\bar{k}$ and a single circumferential mode $m$ are 


$$
\begin{aligned}
& \frac{\partial \rho^{\prime}}{\partial t}+\frac{\partial\left(\rho^{\prime} u_{0}+\rho_{0} u^{\prime}\right)}{\partial x}+\frac{\partial\left(\rho^{\prime} v_{0}+\rho_{0} v^{\prime}\right)}{\partial r} \\
& \quad-\frac{m \rho_{0}}{k r} w_{t}^{\prime}+\frac{\rho^{\prime} v_{0}+\rho_{0} v^{\prime}}{r}=0 \\
& \frac{\partial u^{\prime}}{\partial t}+u_{0} \frac{\partial u^{\prime}}{\partial x}+v_{0} \frac{\partial u^{\prime}}{\partial r}+\left(u^{\prime}+\frac{\rho^{\prime}}{\rho_{0}} u_{0}\right) \frac{\partial u_{0}}{\partial x}+\left(v^{\prime}+\frac{\rho^{\prime}}{\rho_{0}} v_{0}\right) \frac{\partial u_{0}}{\partial r} \\
& \quad+\frac{\partial p^{\prime}}{\rho_{0} \partial x}=0 \\
& \frac{\partial v^{\prime}}{\partial t}+u_{0} \frac{\partial v^{\prime}}{\partial x}+v_{0} \frac{\partial v^{\prime}}{\partial r}+\left(u^{\prime}+\frac{\rho^{\prime}}{\rho_{0}} u_{0}\right) \frac{\partial v_{0}}{\partial x}+\left(v^{\prime}+\frac{\rho^{\prime}}{\rho_{0}} v_{0}\right) \frac{\partial v_{0}}{\partial r} \\
& \quad+\frac{\partial p^{\prime}}{\rho_{0} \partial r}=0 \\
& \frac{\partial w_{t}^{\prime}}{\partial t}+u_{0} \frac{\partial w_{t}^{\prime}}{\partial x}+v_{0} \frac{\partial w_{t}^{\prime}}{\partial r}+\frac{m k}{\rho_{0} r} p^{\prime}+\frac{w_{t}^{\prime} v_{0}}{r}=0
\end{aligned}
$$

where superscript $\left({ }^{\prime}\right)$ and subscript $\left({ }_{0}\right)$ denote perturbation and mean properties, respectively; $u, v$, and $w$ are velocity components in the $x, r$, and $\theta$ directions, respectively; $w_{t}^{\prime}=\partial w^{\prime} / \partial t$. The fluid is modeled as a perfect gas with the homentropic assumption, $p^{\prime}=C_{0}^{2} \rho^{\prime}$, where $C_{0}$ is the sound speed. All variables are nondimensionalized using a reference length $L^{*}$, a reference speed $C_{0}^{*}$, and a reference density $\rho^{*}$. For the numerical examples presented next, these have been taken as $1 \mathrm{~m}, 340 \mathrm{~m} / \mathrm{s}$, and $1.225 \mathrm{~kg} / \mathrm{m}^{3}$.

Compared with the normal LEE implementation in 3-D cylindrical coordinates, the 2.5-D LEE mode is capable of solving spinning mode radiations on an axisymmetric background flowfield without swirl in a 2-D computational domain (the $x-r$ coordinates) that represents huge savings in the computational cost. The spinning modes are introduced in the computation domain as boundary conditions. More details about the implementations and applications of 2.5-D LEE can be found in the literature $[7,9,10,12]$.

For a sheared background flow, in addition to an acoustic mode, both 3-D and 2.5-D LEE models also support a hydrodynamic mode that can develop exponentially to overwhelm the desired acoustic solutions. To suppress the unbounded growth of instabilities, the terms with $\partial u_{0} / \partial r$ were omitted in Eq. (3) for a background flow sheared in the horizontal direction (the $x$ direction) [7,10]. Otherwise, the terms generate numerical discontinuities in the shear layer. The modified equations were termed revised 2.5-D LEE. Although revised 2.5-D LEE are quite similar to Eq. (3), they are given next for the clarity of the paper:

$$
\begin{aligned}
& \frac{\partial \rho^{\prime}}{\partial t}+\frac{\partial\left(\rho^{\prime} u_{0}+\rho_{0} u^{\prime}\right)}{\partial x}+\frac{\partial\left(\rho^{\prime} v_{0}+\rho_{0} v^{\prime}\right)}{\partial r} \\
& \quad-\frac{m \rho_{0}}{k r} w_{t}^{\prime}+\frac{\rho^{\prime} v_{0}+\rho_{0} v^{\prime}}{r}=0 \\
& \frac{\partial u^{\prime}}{\partial t}+u_{0} \frac{\partial u^{\prime}}{\partial x}+v_{0} \frac{\partial u^{\prime}}{\partial r}+\left(u^{\prime}+\frac{\rho^{\prime}}{\rho_{0}} u_{0}\right) \frac{\partial u_{0}}{\partial x}+\frac{\partial p^{\prime}}{\rho_{0} \partial x}=0 \\
& \frac{\partial v^{\prime}}{\partial t}+u_{0} \frac{\partial v^{\prime}}{\partial x}+v_{0} \frac{\partial v^{\prime}}{\partial r}+\left(u^{\prime}+\frac{\rho^{\prime}}{\rho_{0}} u_{0}\right) \frac{\partial v_{0}}{\partial x} \\
& \quad+\left(v^{\prime}+\frac{\rho^{\prime}}{\rho_{0}} v_{0}\right) \frac{\partial v_{0}}{\partial r}+\frac{\partial p^{\prime}}{\rho_{0} \partial r}=0 \\
& \frac{\partial w_{t}^{\prime}}{\partial t}+u_{0} \frac{\partial w_{t}^{\prime}}{\partial x}+v_{0} \frac{\partial w_{t}^{\prime}}{\partial r}+\frac{m k}{\rho_{0} r} p^{\prime}+\frac{w_{t}^{\prime} v_{0}}{r}=0
\end{aligned}
$$

A similar modification was introduced by Bogey et al. to prevent the development of instabilities in LEE [16]. The modification is, however, nonphysical which may affect the overall accuracy of sound solutions. One objective of this work was to find an alternative computational model with clear physical meaning to validate our previous work conducted based on revised 2.5-D LEE.

\section{B. Propagation: Acoustic Perturbation Equations}

Nonacoustic modes, otherwise present in the LEE, are removed in the APE system by applying an acoustic filtering matrix [17]. An indepth discussion of the relevant theoretical background can be found in Ewert and Schröder's work [17], where the APE model was developed from the LEE model in Cartesian coordinates. To extend the APE model for engine applications in cylindrical coordinates, as well as to avoid Hankel transform in the $r-\theta$ coordinates, several extensions and simplifications were made next.

It was assumed that the background mean flow of the cases studied in this work is absent of the discontinuity in the mean flow in the $\theta$ direction. Rather than on the ordinary LEE, an acoustic filtering matrix was constructed based upon the 2.5-D LEE. After applying the matrix on the 2.5-D LEE, vorticity modes that are present in the $x-r$ coordinates are removed. As a result, no hydrodynamic instability is excited in the shear flow considered in this work.

More precisely, by rearranging terms, Eq. (3) takes the following form which is similar to Eqs. $(1,2)$ in Ewert and Schröder's work [17]:

$$
\begin{aligned}
& \frac{\partial \rho^{\prime}}{\partial t}+u_{\infty} \frac{\partial \rho^{\prime}}{\partial x}+\rho_{\infty} \frac{\partial u^{\prime}}{\partial x}+\rho_{\infty} \frac{\partial v^{\prime}}{\partial r}=S_{1} \\
& \frac{\partial u^{\prime}}{\partial t}+u_{\infty} \frac{\partial u^{\prime}}{\partial x}+\frac{\partial p^{\prime}}{\rho_{\infty} \partial r}=S_{2} \\
& \frac{\partial v^{\prime}}{\partial t}+u_{\infty} \frac{\partial v^{\prime}}{\partial x}+\frac{\partial p^{\prime}}{\rho_{\infty} \partial r}=S_{3} \\
& \frac{\partial w_{t}^{\prime}}{\partial t}+u_{\infty} \frac{\partial w_{t}^{\prime}}{\partial x}=S_{4}
\end{aligned}
$$

where $\rho_{\infty}$ and $u_{\infty}$ are the constant density and constant velocity in the far field; the terms $S_{1}-S_{4}$ include acoustic modes, vorticity modes, and several terms with $1 / r$, whose analytical counterparts, through the Fourier transform, cannot be expressed straightforwardly. The Fourier and Laplace transforms can be applied to construct an acoustic filtering matrix that thereafter isolates nonacoustic modes in the $x-r$ coordinates from $S_{1}-S_{4}$. It is worth emphasizing that the Fourier transform is conducted on Eq. (5) in the $x-r$ coordinates, rather than in the whole cylindrical $(x-r-\theta)$ coordinates. Along with the absence of $1 / r$ on the left-hand side of Eq. (5), a Hankel transform normally used for the cylindrical coordinates can be avoided. The price to pay is that the simplified operation cannot remove nonacoustic modes of the $\theta$ direction, which contribute little to the instability development in the interested applications for the absence of the mean flow discontinuity in the $\theta$ direction. More precisely, the Fourier transform defined in the $x-r$ coordinates is

$$
\tilde{\mathbf{F}}(\alpha, \beta)=\int_{-\infty}^{\infty} \int_{-\infty}^{\infty} f(x, r) H(r) e^{-i(\alpha x+\beta r)} \mathrm{d} x \mathrm{~d} r
$$

The wave numbers $\alpha$ and $\beta$ are related to Fourier transforms in the $x$ and $r$ coordinates, respectively. $H(r)$ is the Heaviside function that is used to limit the integral of the Fourier transform into the positive half-plane in the $r$ direction. The variable $f$ can be $\rho, u, v$, or $w_{t}$. The imaginary unit $i$ is associated with the Fourier transform. Equation (5) is subsequently transformed to the following equation in the vector form:

$$
\mathbf{A} \tilde{\mathbf{U}}=\tilde{\mathbf{S}}
$$

where $\tilde{\mathbf{U}}=\left(\tilde{\rho}, \tilde{u}, \tilde{v}, \tilde{w}_{t}\right)$ represents the Fourier/Laplace transform pairs of the primitive variables, and $\tilde{\mathbf{S}}$ denotes the Fourier/Laplace transform pairs for $\left(S_{1}, S_{2}, S_{3}, S_{4}\right)$ of Eq. (5). The operator matrix A is

$$
\left(\begin{array}{cccc}
s-u_{\infty} \alpha & -\rho_{\infty} \alpha & -\rho_{\infty} \beta & 0 \\
-\alpha / \rho_{\infty} & s-u_{\infty} \alpha & 0 & 0 \\
-\beta / \rho_{\infty} & 0 & s-u_{\infty} \alpha & 0 \\
0 & 0 & 0 & s-u_{\infty} \alpha
\end{array}\right)
$$


where the complex number $s$ is associated with the Laplace transform. The eigenvalues of $\mathbf{A}$ are $\lambda_{1}=s-u_{\infty} \alpha+\sqrt{\alpha^{2}+\beta^{2}}$, $\lambda_{2}=s-u_{\infty} \alpha-\sqrt{\alpha^{2}+\beta^{2}}$, and $\lambda_{3}=\lambda_{4}=s-u_{\infty} \alpha$. The corresponding eigenvectors are

$$
\begin{array}{cc}
x_{1}=\left(\begin{array}{c}
1 \\
\frac{-\alpha}{\rho_{\infty}\left(\alpha^{2}+\beta^{2}\right)^{1 / 2}} \\
\frac{-\beta}{\rho_{\infty}\left(\alpha^{2}+\beta^{2}\right)^{1 / 2}}
\end{array}\right), & x_{2}=\left(\begin{array}{c}
\frac{\alpha}{\rho_{\infty}\left(\alpha^{2}+\beta^{2}\right)^{1 / 2}} \\
\frac{\beta}{\rho_{\infty}\left(\alpha^{2}+\beta^{2}\right)^{1 / 2}} \\
0
\end{array}\right) \\
x_{3}=\left(\begin{array}{c}
0 \\
1 \\
\frac{-\alpha}{\beta} \\
0
\end{array}\right), & x_{4}=\left(\begin{array}{l}
0 \\
0 \\
0 \\
1
\end{array}\right)
\end{array}
$$

It is easy to see that only $x_{3}$ is associated with the vorticity mode in the $x-r$ coordinates. The corresponding acoustic filtering matrix [17] is $\mathbf{T}^{a}=x_{1}\left(x_{1}^{-1}\right)^{T}+x_{2}\left(x_{2}^{-1}\right)^{T}+x_{4}\left(x_{4}^{-1}\right)^{T}$, where $T$ denotes the transpose of a matrix. As a result, the acoustic filtering matrix for Eq. (5) in the $x-r$ coordinates is

$$
\mathbf{T}^{a}=\left(\begin{array}{cccc}
1 & 0 & 0 & 0 \\
0 & \frac{\alpha^{2}}{\sqrt{\alpha^{2}+\beta^{2}}} & \frac{\alpha \beta}{\sqrt{\alpha^{2}+\beta^{2}}} & 0 \\
0 & \frac{\alpha \beta}{\sqrt{\alpha^{2}+\beta^{2}}} & \frac{\beta^{2}}{\sqrt{\alpha^{2}+\beta^{2}}} & 0 \\
0 & 0 & 0 & 1
\end{array}\right)
$$

Applying $\mathbf{T}^{a}$ on $\tilde{\mathbf{S}}$ of Eq. (7) yields

$$
\left(\begin{array}{c}
\tilde{S}_{1}^{a} \\
\tilde{S}_{2}^{a} \\
\tilde{S}_{3}^{a} \\
\tilde{S}_{4}^{a}
\end{array}\right)=\left(\begin{array}{cccc}
1 & 0 & 0 & 0 \\
0 & \frac{\alpha^{2}}{\sqrt{\alpha^{2}+\beta^{2}}} & \frac{\alpha \beta}{\sqrt{\alpha^{2}+\beta^{2}}} & 0 \\
0 & \frac{\alpha \beta}{\sqrt{\alpha^{2}+\beta^{2}}} & \frac{\beta^{2}}{\sqrt{\alpha^{2}+\beta^{2}}} & 0 \\
0 & 0 & 0 & 1
\end{array}\right)\left(\begin{array}{c}
\tilde{S}_{1} \\
\tilde{S}_{2} \\
\tilde{S}_{3} \\
\tilde{S}_{4}
\end{array}\right)
$$

The Fourier/Laplace transform pairs $\left(\tilde{S}_{1}, \tilde{S}_{2}, \tilde{S}_{3}, \tilde{S}_{4}\right)$ in Eq. (7) are subsequently replaced with $\left(\tilde{S}_{1}^{a}, \tilde{S}_{2}^{a}, \tilde{S}_{3}^{a}, \tilde{S}_{4}^{a}\right)$. After applying the inverse Fourier and the inverse Laplace transforms, it is easy to see that the first and fourth equations in Eqs. (3-5) remain the same by $\mathbf{T}_{a}$, which only works on the momentum equations in the $x$ and $r$ coordinates. In addition, the subsequent processing is similar to the processing in the $x-y$ coordinates, which was thoroughly discussed in Ewert and Schröder's work [17], leading to four sets of APE equations: APE-1 to APE-4 systems. This work follows the APE-2 system, where the momentum equations have the form $\partial \mathbf{u}^{\prime} / \partial t+$ $\nabla\left(\mathbf{u}_{0} \cdot \mathbf{u}^{\prime}\right)+\nabla\left(p^{\prime} / \rho_{0}\right)=\mathbf{S} \quad[17], \quad \mathbf{u}^{\prime}=\left(u^{\prime}, v^{\prime}\right), \quad \mathbf{u}_{0}=\left(u_{0}, v_{0}\right)$, $\mathbf{S}=\left(S_{2}^{a}, S_{3}^{a}\right)$. The whole APE extended for the duct applications investigated in this work are listed next:

$$
\begin{gathered}
\frac{\partial \rho^{\prime}}{\partial t}+\frac{\partial\left(\rho^{\prime} u_{0}+\rho_{0} u^{\prime}\right)}{\partial x}+\frac{\partial\left(\rho^{\prime} v_{0}+\rho_{0} v^{\prime}\right)}{\partial r} \\
-\frac{m \rho_{0}}{k r} w_{t}^{\prime}+\frac{\rho^{\prime} v_{0}+\rho_{0} v^{\prime}}{r}=0 \\
\frac{\partial u^{\prime}}{\partial t}+\frac{\partial\left(u_{0} u^{\prime}+v_{0} v^{\prime}\right)}{\partial x}+\frac{\left(\partial p^{\prime} / \rho_{0}\right)}{\partial x}=S_{2}^{a} \\
\frac{\partial v^{\prime}}{\partial t}+\frac{\partial\left(u_{0} u^{\prime}+v_{0} v^{\prime}\right)}{\partial r}+\frac{\left(\partial p^{\prime} / \rho_{0}\right)}{\partial r}=S_{3}^{a} \\
\frac{\partial w_{t}^{\prime}}{\partial t}+u_{0} \frac{\partial w_{t}^{\prime}}{\partial x}+v_{0} \frac{\partial w_{t}^{\prime}}{\partial r}+\frac{m k}{\rho_{0} r} p^{\prime}+\frac{w_{t}^{\prime} v_{0}}{r}=0
\end{gathered}
$$

$S_{2}^{a}$ and $S_{3}^{a}$ are the sound sources due to the existence of a shear flow. Other definitions of variables were already provided after Eq. (3). The main objective of this work was to study the spinning mode propagation in the near field, refractions in the sheared flow, and radiation to the far field. Mean flow sound source $S_{2}^{a}$ and $S_{3}^{a}$ were not considered and set to zero in the current computation. The same processing can be found in the first test case of Ewert and Schröder's work [17]. It is also worth noting that the homogeneous APE without any source terms are actually perturbation equations introduced by
Pierce [19]. The equations can be properly applied only in the highfrequency regime, where the characteristic scales of the perturbation are much smaller than the corresponding scales of the background flow. The assumption is valid for the situation described in this work; for example, the frequency of the spinning mode wave is $633 \mathrm{~Hz}(k$ is scaled to 11.7) for the following canonical case and $1532 \mathrm{~Hz}$ ( $k$ is scaled to 28.3) for the following realistic engine case.

\section{Spinning Modes}

A single spinning mode $(m, n)$ was studied. The incident wave assigned in the acoustic entry area is defined as

$$
\begin{aligned}
& \rho^{\prime}(x, r, \theta, t)=a\left[J_{m}\left(k_{r} r\right)+c_{1} Y_{m}\left(k_{r} r\right)\right] \cos \left(k t-k_{a} x-m \theta\right) \\
& u^{\prime}(x, r, \theta, t)=\frac{k_{a}}{k-k_{a} M_{j}} \rho^{\prime} \\
& v^{\prime}(x, r, \theta, t)=-\frac{a}{k-k_{a} M_{j}} \frac{d\left[J_{m}\left(k_{r} r\right)+c_{1} Y_{m}\left(k_{r} r\right)\right]}{d r} \\
& \times \sin \left(k t-k_{a} x-m \theta\right) \\
& w_{t}^{\prime}(x, r, \theta, t)=-\frac{a m k\left[J_{m}\left(k_{r} r\right)+c_{1} Y_{m}\left(k_{r} r\right)\right]}{r\left(k-k_{a} M_{j}\right)} \\
& \times \sin \left(k t-k_{a} x-m \theta\right) \\
& w^{\prime}(x, r, \theta, t)=\frac{m \rho^{\prime}}{r\left(k-k_{a} M_{j}\right)}
\end{aligned}
$$

where $M_{j}$ is the Mach number inside the entry area of a duct. $J_{m}$ and $Y_{m}$ are the $m$ th order Bessel functions of the first and second kind, respectively. The value of $a$ is fixed at $10^{-4}$ to 1 ) test the low dissipation of the high-order schemes [6] employed in our numerical implementation, and 2) ensure small relative changes for acoustic modes compared with nondimensionalized density (as required for LEE and APE). A common practice to normalize the acoustic modes, however, is in terms of either their acoustic power or the pressure at the duct wall. The axial wave number is $k_{a}$, and $k_{r}$ is the radial wave number. The $n$th solution $k_{r}$ of the following equation is determined by the hard-wall boundary conditions of the duct:

$$
\frac{\mathrm{d}\left[J_{m}\left(y_{\text {outer }} k_{r}\right)\right]}{\mathrm{d} r} \frac{\mathrm{d}\left[Y_{m}\left(y_{\text {inner }} k_{r}\right)\right]}{\mathrm{d} r}-\frac{\mathrm{d}\left[J_{m}\left(y_{\text {inner }} k_{r}\right)\right]}{\mathrm{d} r} \frac{\mathrm{d}\left[Y_{m}\left(y_{\text {outer }} k_{r}\right)\right]}{\mathrm{d} r}=0
$$

where $y_{\text {outer }}$ and $y_{\text {inner }}$ are the bypass duct inner wall radius and the inner hub radius. The axial wave number $k_{a}$ is calculated from

$$
k_{a}=\frac{k}{1-M_{j}^{2}}\left(-M_{j} \pm \sqrt{1-\frac{k_{r}^{2}\left(1-M_{j}^{2}\right)}{k^{2}}}\right)
$$

the selection of plus or minus $( \pm)$ signs in the parentheses is determined by the propagation direction of the spinning wave, for example, plus $(+)$ is for the positive propagation direction in the axial coordinate, and vice versa. The constant $c_{1}$ satisfies the following relations:

$$
c_{1}=-\frac{(\mathrm{d} / \mathrm{d} r)\left[J_{m}\left(y_{\text {outer }} k_{r}\right)\right]}{(\mathrm{d} / \mathrm{d} r)\left[Y_{m}\left(y_{\text {outer }} k_{r}\right)\right]}
$$

or

$$
c_{1}=-\frac{(\mathrm{d} / \mathrm{d} r)\left[J_{m}\left(y_{\text {inner }} k_{r}\right)\right]}{(\mathrm{d} / \mathrm{d} r)\left[Y_{m}\left(y_{\text {inner }} k_{r}\right)\right]}
$$

\section{Radiation}

An integral form of the Ffowcs Williams and Hawkings (FW-H) equation was solved numerically to allow the near- and far-field noise levels to be determined efficiently. The FW-H formulation is attractive in comparison to other integral methods as it permits the passage of hydrodynamic disturbances through an FW-H integral 


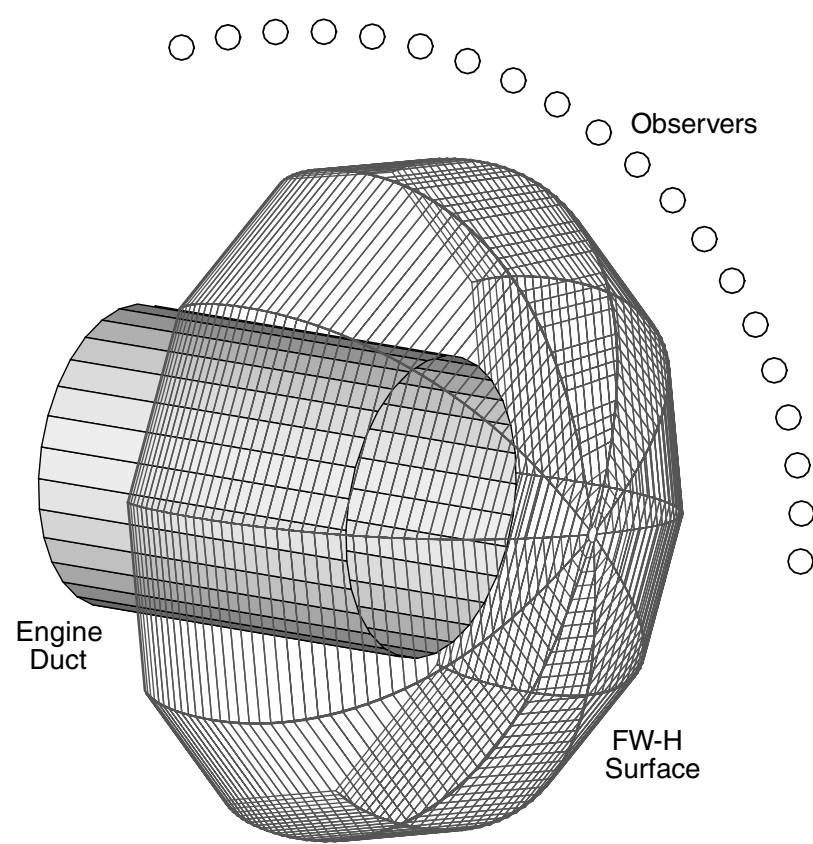

Fig. 2 Three-dimensional FW-H integral surface around engine duct.

surface without affecting the acoustic field. The particular timedomain integral solution implemented for this work is known as formulation 1A following Farassat and Succi [23].

A 3-D integral surface needs to be constructed to evaluate the farfield noise level. Figure 2 shows a three-dimensional FW-H integral surface that was constructed around the engine duct. In the numerical implementation, the FW-H integral surface was divided into panels. The full 3-D data on the panels were generated from either the LEE or the APE solution by expanding the Fourier series representation in the circumferential direction, using Eq. (2), that is, $p^{\prime}(\theta, t)=p^{\prime}(0, t-m \theta / k)$. The 3-D solutions were provided to the FW-H solver, along with the geometry information of the integral surface, to predict the far-field directivity. The surface integration was approximated on each panel by midpanel quadrature [24]. The FW-H implementation was previously validated with analytic solutions to the problem of acoustic radiation from a circular unflanged duct $[9,10]$. Comparisons were also made with a finite element/infinite element code for the case of acoustic radiation from an engine intake with a realistic background flow [12]. It was found that the larger the panel number, the better agreement that could be obtained in the interference dip angles. For the computation of the $m$ th circumferential mode, a total of $m \times N \times G$ patches in the circumferential direction are required for the accuracy, where $N$ is time steps per modal period, and $G$ is the grid number that is cut through by the surface. The far-field observers are generally located at 100 units from the duct.

\section{E. Numerical Schemes}

The numerical solver implemented for this work employed a sixthorder prefactored compact scheme [25] to calculate spatial derivatives. A fourth-order 4/6-stage explicit Runge-Kutta scheme [26] was used for time integration. A slip-wall boundary condition was used for the hard duct walls. Absorbing zones [11] were placed around the boundary of the physical computational domain to provide nonreflecting boundary conditions. Inside the duct, an absorbing zone, with a width of at least one acoustic wavelength, was used to absorb any spurious numerical waves, as well as acting as a spinning mode input region with the boundary conditions [Eq. (13)].

\section{Noise Radiation from Semi-Infinite Duct}

\section{A. Setup}

A canonical test case of modal radiation from a subsonic jet issuing into a stationary flowfield, described by Munt [15], was first

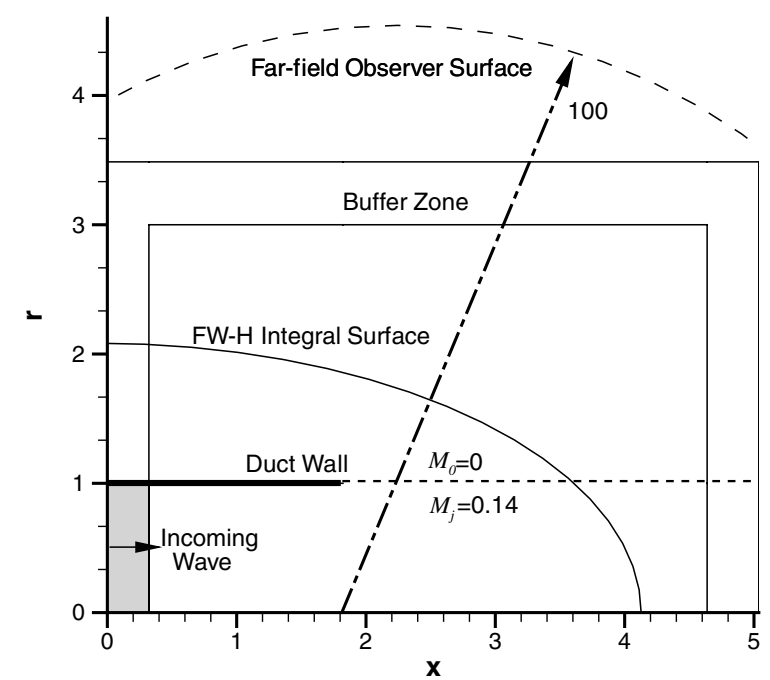

Fig. 3 Schematic of nondimensionalized problem setup including semiinfinite duct geometry and subsonic jet flow. Far-field distance is not scaled.

investigated with the LEE and APE models. In Fig. 3, the problem setup is depicted. The background flow is illustrated in terms of the Mach number, which is set to $0.14\left(M_{j}\right)$ inside the subsonic jet, and zero $\left(M_{0}\right)$ at the other field. The axial velocity $u_{0}$ in the $x$ direction is $C_{0} M_{j}$ inside the jet or zero outside the jet. The radial velocity $v_{0}$ in the $r$ direction is zero. The nondimensionalized radius of the duct and the other nondimensionalized mean flow variables, density $\rho_{0}$ and pressure $p_{0}$, are 1 unit. The thickness of the duct is assumed to be infinitely thin. The lip of the duct is at $(1.0,1.8)$. Inside the duct, a buffer zone [11] was used to absorb the reflective spurious waves, as well as to accommodate incoming modal waves, which were already defined in Eq. (13). Another buffer zone was placed around the outer boundaries of the computational domain. The target solutions of this buffer zone are set to zero to absorb spurious numerical reflections. The far-field directivity from Munt's analytical solution [15] was used in validation, but its amplitude was scaled to that used by Homicz and Lordi [27] so that the results could also be compared in the situation of a uniform background flow. Hence, in terms of comparison with the Munt's solutions [15], only the radiation peak angle, the shadow interference dip angle, and the dynamic range of the pressure were compared.

\section{B. Results and Discussion}

Figure $\underline{4}$ shows that, by using 2.5-D LEE [Eq. (3)], numerical instabilities are generated in the shear layer. A similar profile of the instabilities can also be found in the literature [16] by using LEE for sound waves passing through a shear layer. The instabilities will

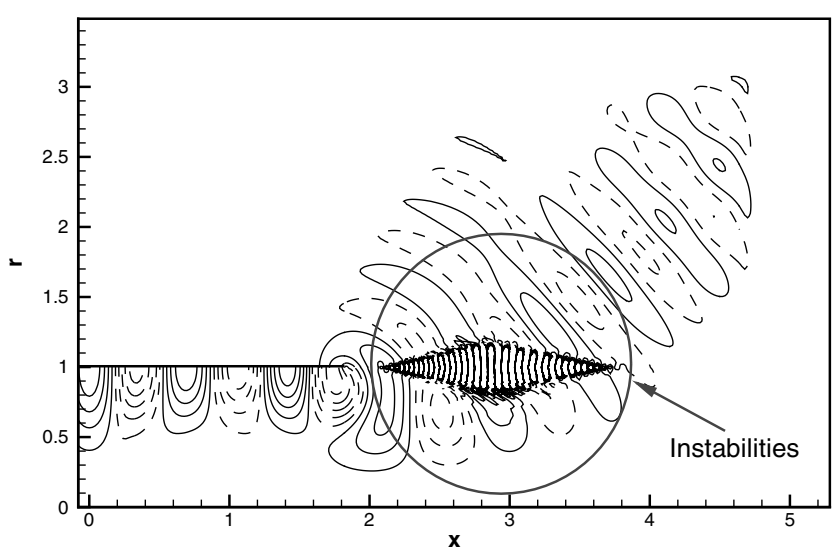

Fig. 4 Numerical instabilities developed in shear layer outside of semiinfinite duct. 


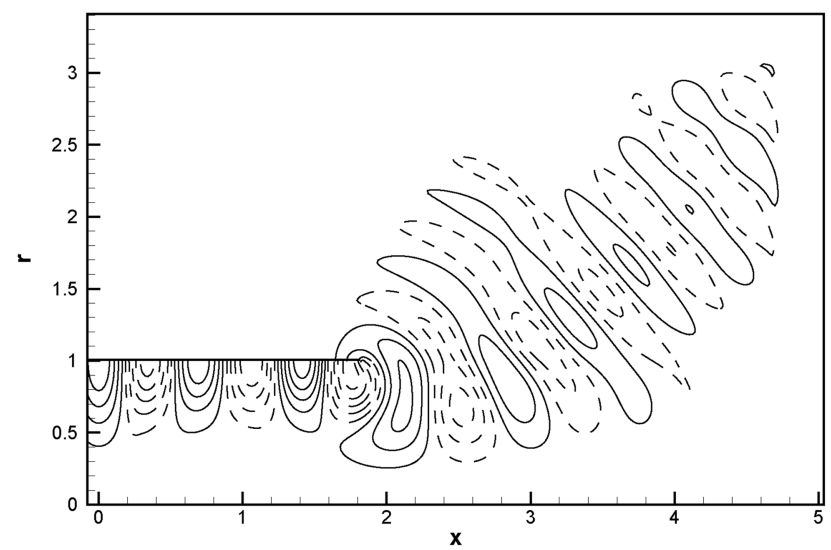

a) Pressure contours solved by revised 2.5-D LEE

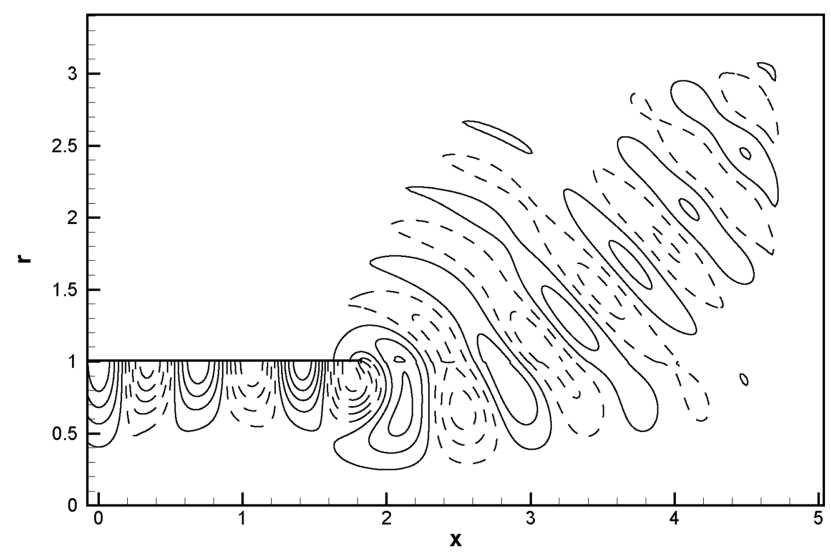

c) Pressure contours solved by APE

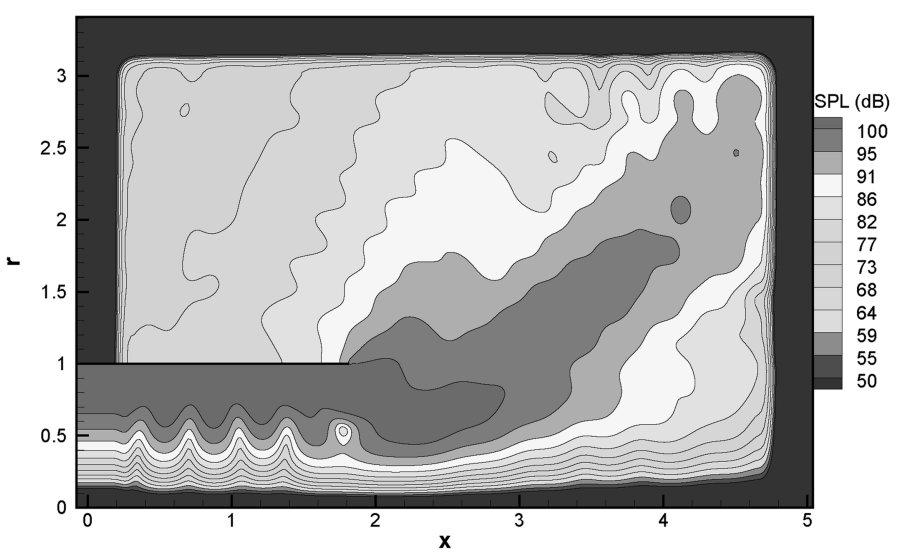

b) SPL solved by revised 2.5-D LEE

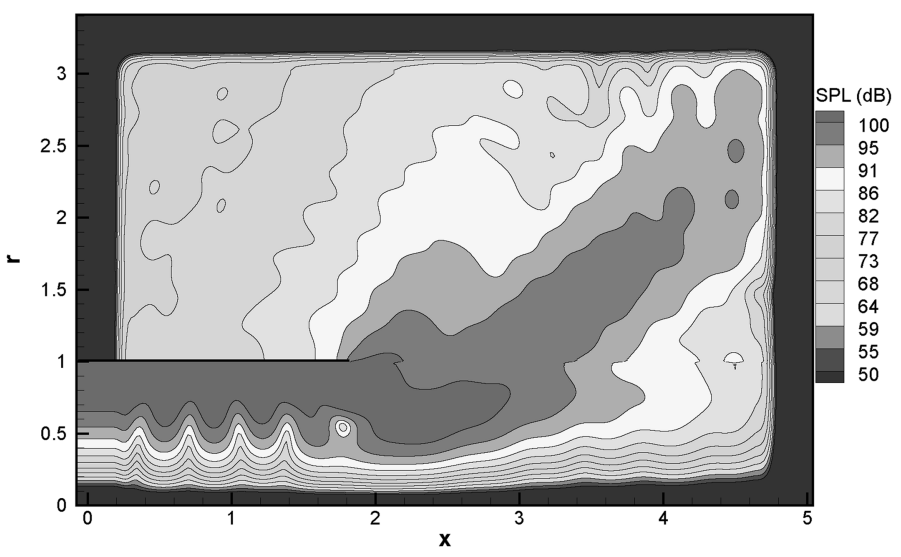

d) SPL solved by APE

Fig. 5 Pressure perturbation and SPL contours of single spinning mode wave from semi-infinite duct, where $m=4, n=1, k=11.7$, and 10 pressure perturbation contours between $\pm 4 e^{-5}$ are displayed.

develop exponentially to cover the whole computational domain and can overwhelm the desired spinning mode solutions quickly. To address the problem, instead of the original 2.5-D LEE, the revised 2.5-D LEE [Eq. (4)] and APE [Eq. (12)] were used. Figure 5 compares the sound pressure perturbation and sound pressure level (SPL) predicted by the two models, where SPL $=20 \log _{10}\left[p_{\text {rms }}^{\prime} /\right.$ $\left(2 \times 10^{-5}\right)$ ]. The variable of $p_{\text {rms }}^{\prime}$ is the root mean square of $p^{\prime}$. Figure 5 shows that the numerical instabilities were removed by using either revised 2.5-D LEE or APE. The near-field pressure perturbation and the SPL contours from both models are similar. The

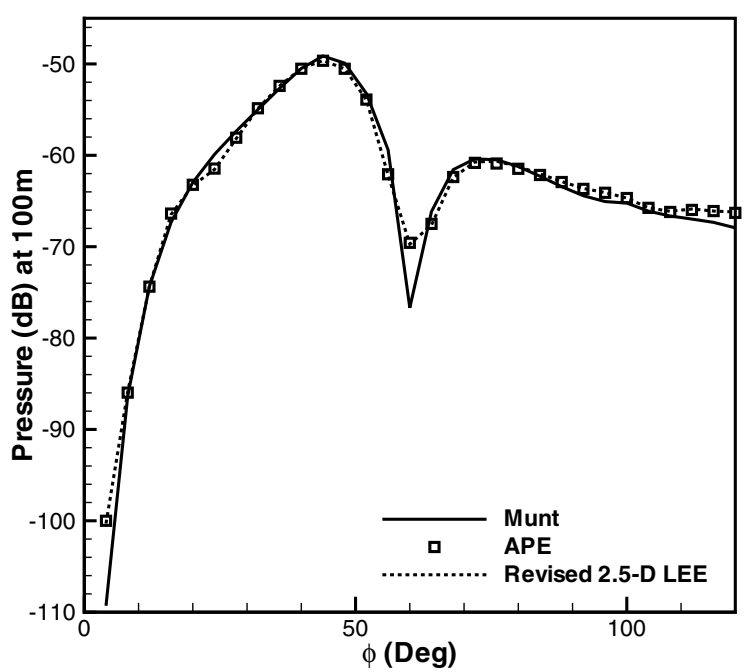

Fig. 6 Far-field directivity of semi-infinite duct radiation. perturbed SPL results are caused by spurious reflections, mainly from the top right corner of the computational domain. The negative effect of the numerical reflections over the far-field prediction can be reduced by using either a larger computational domain or an irregular computational domain with a boundary normal to the sound propagation direction. From the placement position of the FW-H surface, it can be seen that most spurious reflections only affect farfield results above $90 \mathrm{deg}$. The perturbation was further reduced by providing several periods of data of sound propagation to the $\mathrm{FW}-\mathrm{H}$ solver to smooth the prediction output.

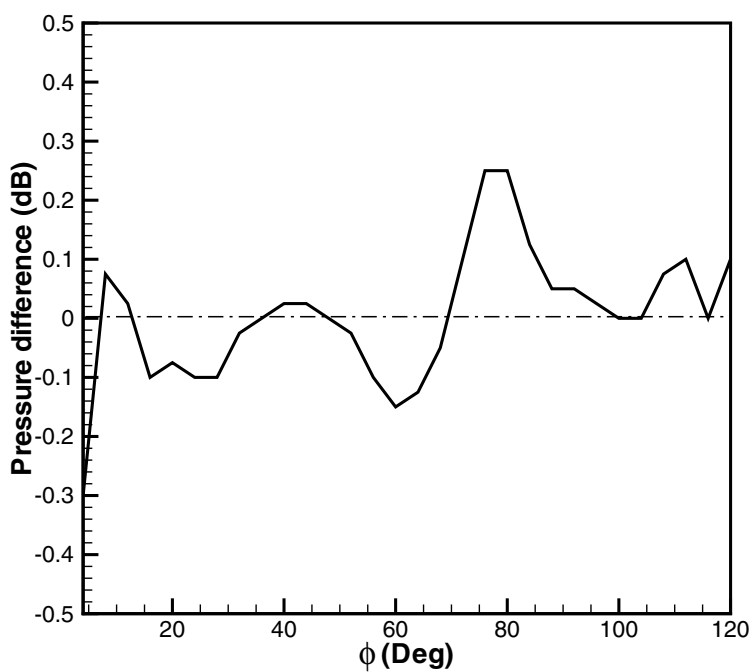

Fig. 7 Directivity difference between revised 2.5-D LEE model and APE model for semi-infinite duct problem. 


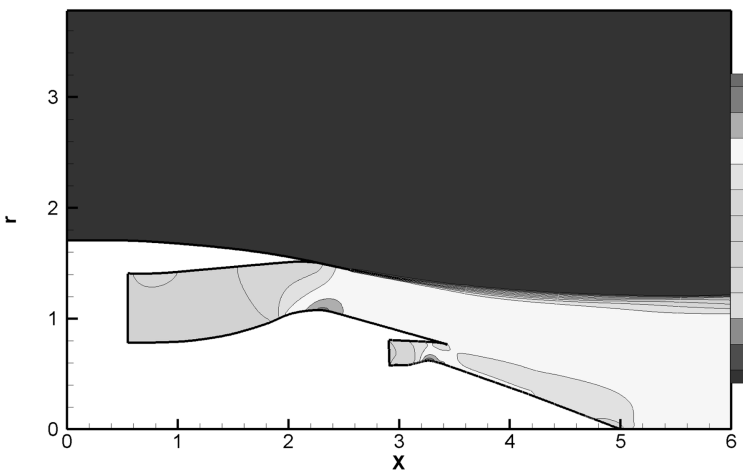

a) Mean mach number distribution

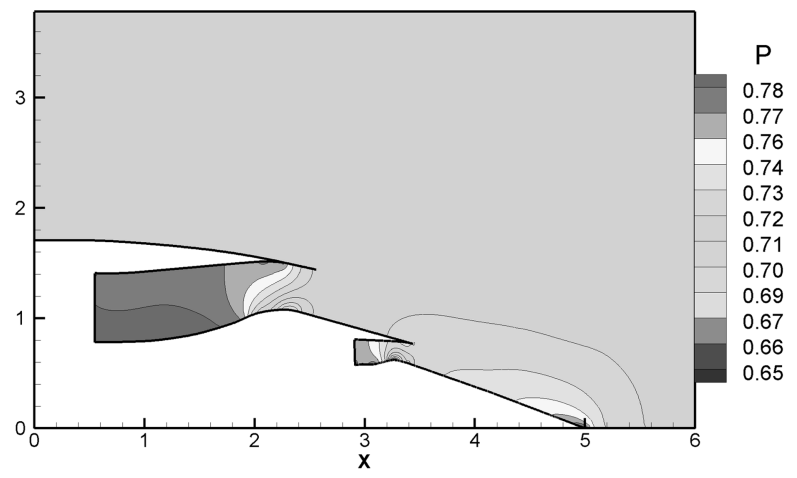

b) Nondimensionalized pressure distribution

Fig. 8 Background flowfield of generic test case.

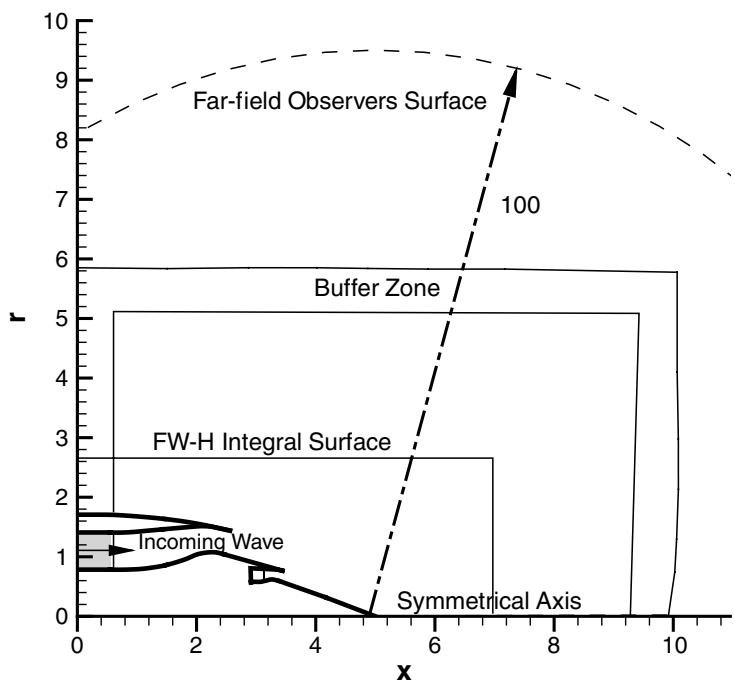

Fig. 9 Nondimensionalized problem setup including aircraft engine bypass and exhaust geometry, displayed by thick lines. Far-field distance is not scaled.

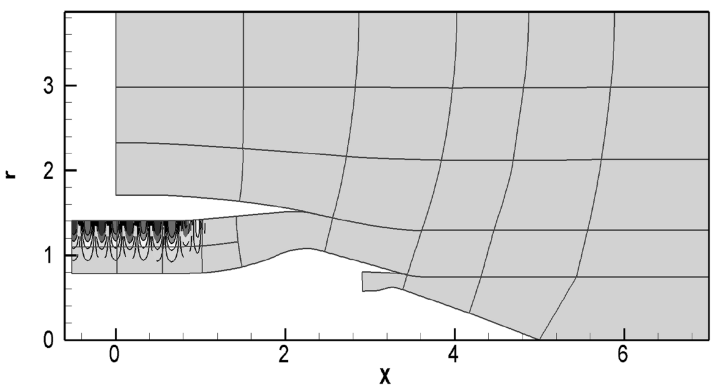

a) $t=1$

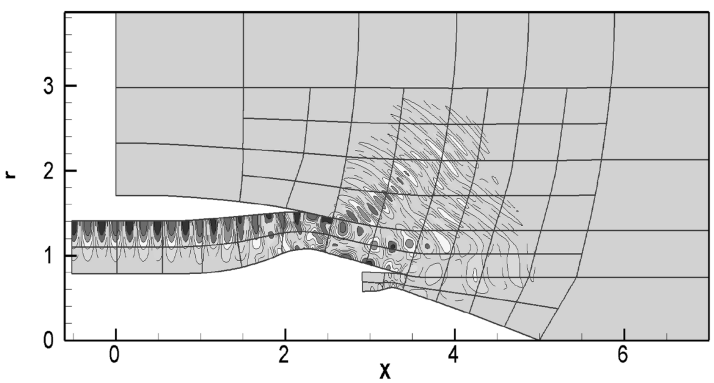

c) $t=4$
The far-field pressure directivities are compared with the asymptotic solution [15] in Fig. 6, which shows that the predictions of revised 2.5-D LEE and APE agree well with the asymptotic solution. Figure 7 compares the difference between the directivities obtained with the revised 2.5-D LEE and the APE models. Results show that there is little difference. It should be noted that, for this canonical test case, and considering $v_{0}=0$ and $\partial u_{0} / \partial x=0$, the two models [Eqs. (4) and (12)] are only different in the momentum equation in the $r$ direction. The different terms are $u_{0}\left(\partial v^{\prime} / \partial x-\partial u^{\prime} / \partial r\right)-u^{\prime} \partial u_{0} / \partial r$. Because the velocity field for sound waves is irrotational, the difference can be simplified to $-u^{\prime} \partial u_{0} / \partial r$ for acoustic solutions. The little difference between two sets of equations, obviously, only happens for the current case with the idealized background flow. To validate the revised 2.5-D LEE with APE, it will be more significant to consider general cases with a realistic background flow, where the two models should have considerably different forms in both $x$ and $r$ momentum equations.

\section{Noise Radiation from Aircraft Engine Bypass Duct}

\section{A. Setup}

The geometries given in Figs. 8 and 9 come from a generic engine bypass duct. The length scale was nondimensionalized by $L^{*}$. The inflow boundary geometries are as follows: the inner wall radius of the core exhaust duct is 0.8 , the exhaust cone radius is 0.57 ,

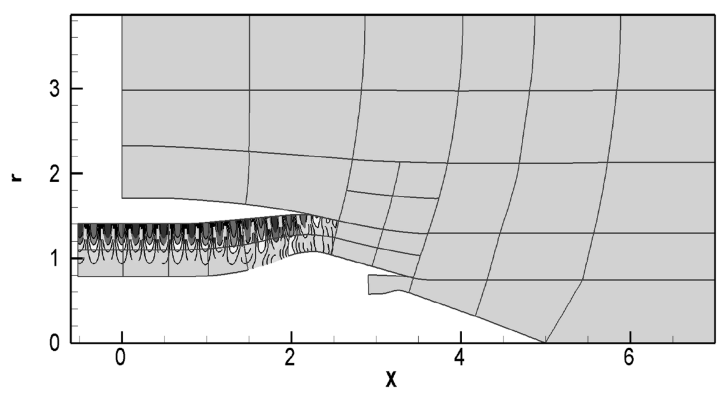

b) $t=2$

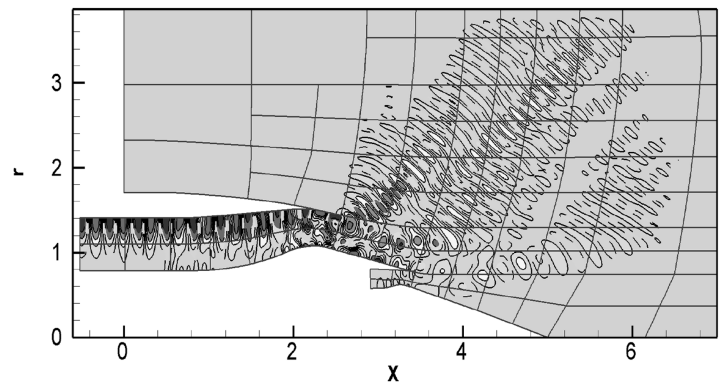

d) $t=6$

Fig. 10 Evolution of adaptively refined mesh with propagation of acoustic waves from engine exhaust. Gray lines represent block boundaries of adaptively refined mesh. Ten contour levels are used between $\pm 2 e^{-5}$. 
Table 1 Adaptive mesh refinement computation time

\begin{tabular}{lcccc}
\hline \hline Grids/time & $t=1$ & $t=2$ & $t=5$ & $t=8$ \\
\hline Adaptive mesh & $1478 \mathrm{~s}$ & $3401 \mathrm{~s}$ & $13,460 \mathrm{~s}$ & $26,670 \mathrm{~s}$ \\
Uniform mesh & $3971 \mathrm{~s}$ & $8002 \mathrm{~s}$ & $20,120 \mathrm{~s}$ & $31,920 \mathrm{~s}$ \\
\hline \hline
\end{tabular}

$y_{\text {outer }}=1.4$, and $y_{\text {inner }}=0.8$. The background flow of the generic bypass duct test case was determined from the solution of the Reynolds-averaged Navier-Stokes with k- $\omega$ turbulence model using the FLUENT commercial flow solver. The duct inflow pressure condition is $112,058 \mathrm{~Pa}$. The pressure outlet boundary condition is $101,320 \mathrm{~Pa}$. The fluid is modeled as a perfect gas with the homentropic assumption. Temperature is $299.2 \mathrm{~K}$. For simplicity, the axial velocity is $115 \mathrm{~m} / \mathrm{s}$ in both the bypass duct flow entry area and the core exhaust flow entry area. The exhaust stream is issued into a stationary environment. The background flow in the vicinity of the engine is illustrated in Fig. $\underline{8}$ in terms of the Mach number contours and nondimensionalized pressure contours. The reference speed is $C_{0}^{*}$. The reference pressure is $\rho^{*} C_{0}^{* 2}$. For the present test case, the mean flow conditions in the core exhaust duct are the same as those in the bypass duct. The main features of the flow are the regions of flow acceleration near the bypass and core exhaust exits and the shear layer between the bypass exhaust and stationary environment. The viscous boundary layers on the engine surfaces were removed before the spinning mode acoustic calculations by extrapolating the inner flow solution to the surface. The only viscous features resolved in the acoustic computations, therefore, were the bypass and core shear layers. The setup of buffer zones resembles the previous case study.

The computational grid is a structured multiblock grid. The grid comprises a total of 180,000 grid points. The grid point distribution is such that the lowest grid resolution within the domain is approximately above the minimum grid resolution of 12 points per

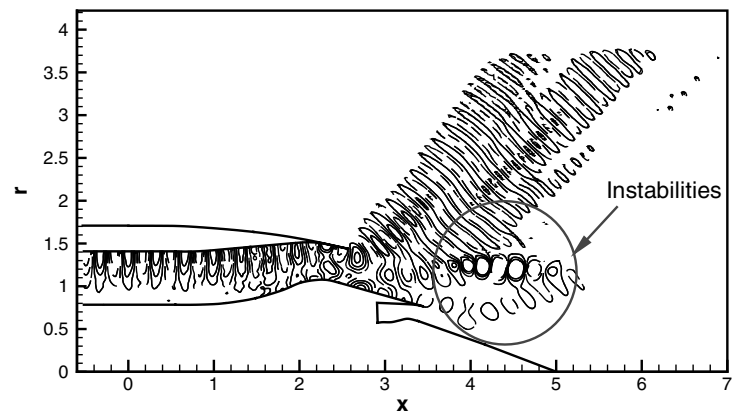

a) Solution by 2.5-D LEE, $t=12$

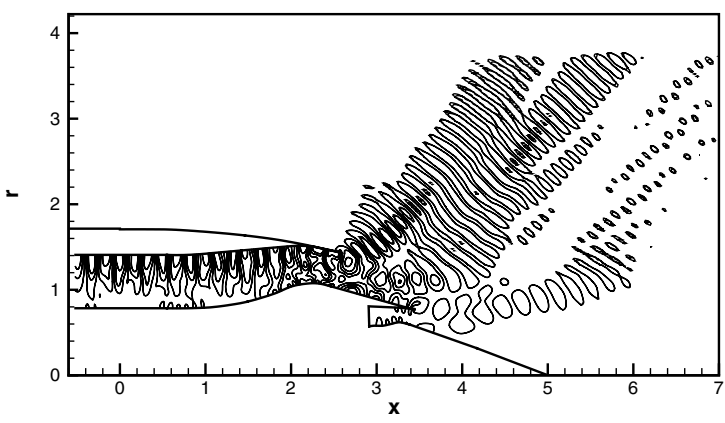

b) Solution by APE, $t=20$

Fig. 11 Perturbation pressure contours computed by 2.5-D LEE [Eq. ( $\underline{3}$ )] and APE [Eq. ( $\underline{\text { 12) }}$ ], where $m=13, n=1, k=28.3$. Ten contour levels are used between $\pm 2 e^{-5}$.

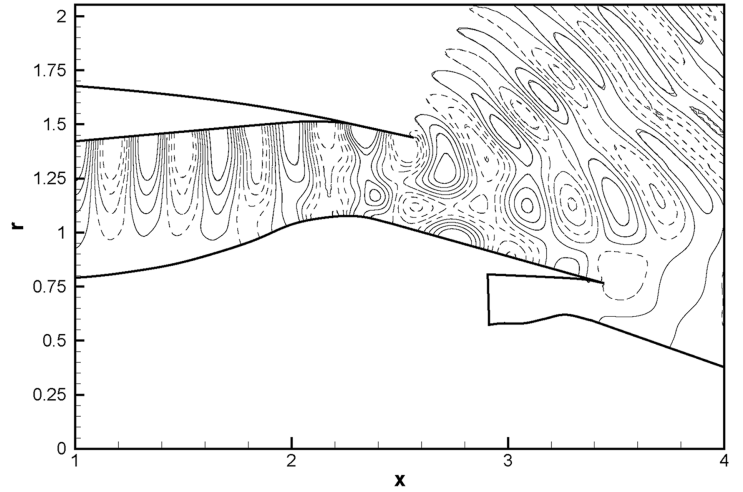

a) Pressure contour solved by revised 2.5-D LEE

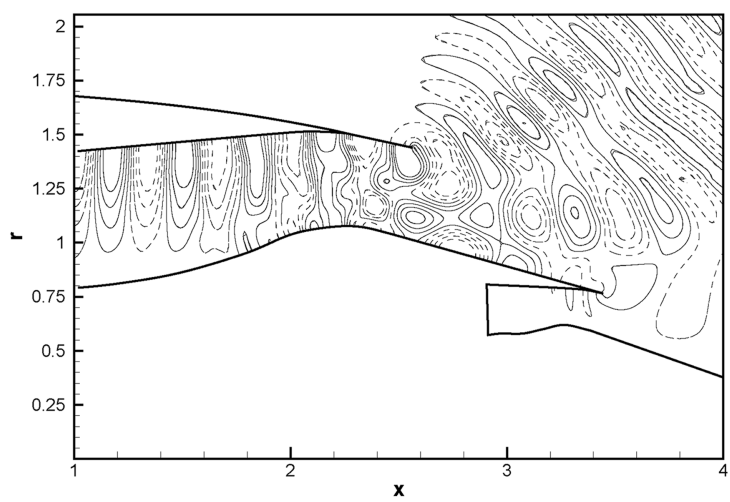

c) Pressure contour solved by APE

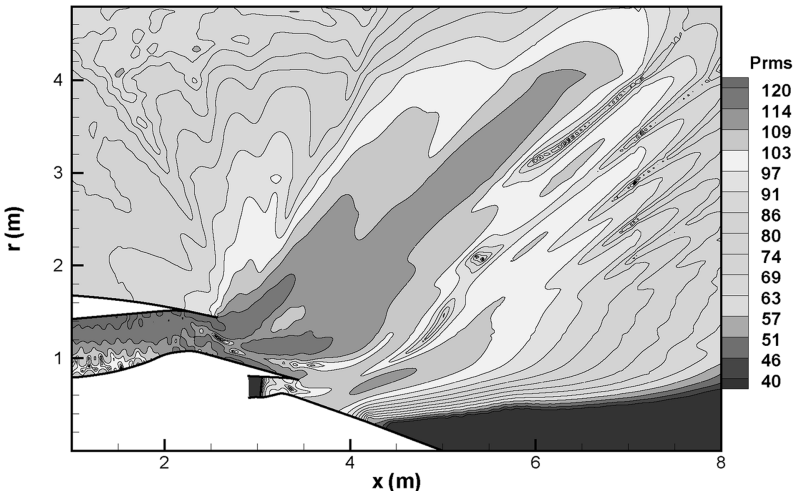

b) SPL solved by revised 2.5-D LEE

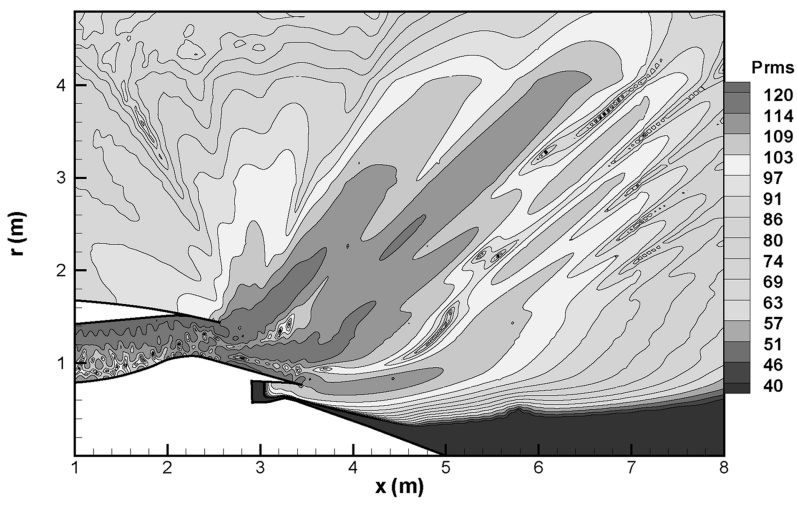

d) SPL solved by APE

Fig. 12 APE prediction of perturbation pressure and SPL contours of several single spinning mode waves, where $m=13, n=1, k=28.3$. Ten contour levels between $\pm 2 e^{-5}$ are displayed. 
wavelength that was found to be necessary for accurate computations. To accelerate the computational speed, the technique of AMR was used.

\section{B. Adaptive Mesh Refinement}

In an earlier work [28], a block-structured AMR code was constructed and tested against benchmark problems on rectangular meshes. The code was extended to work on a distributed-memory parallel machine using a message passing interface library and supported body-fitted meshes to solve aeroacoustic problems of practical significance, for example, acoustic radiation from a general aeroengine intake [12]. Spectral analysis proved the stability of the employed high-order spatial schemes on an adaptively refined mesh [28]. The pseudospectra method [29] could analyze transient stability under an AMR environment. More details about the employed AMR algorithm, its flexibility, efficiency, numerical properties, and parallel performance have been addressed by Huang et al. [12].

It was found that a Cartesian mesh with a low-order immersed boundary method [30] performed more poorly than a body-fitted mesh to solve sound propagation problems with curved geometries [12]. There were already some other attempts to use the AMR technique for body-fitted multiblock meshes [21,31], where curved geometries were allowed to be transformed into and simulated using a uniform computational domain. This can be achieved by using the

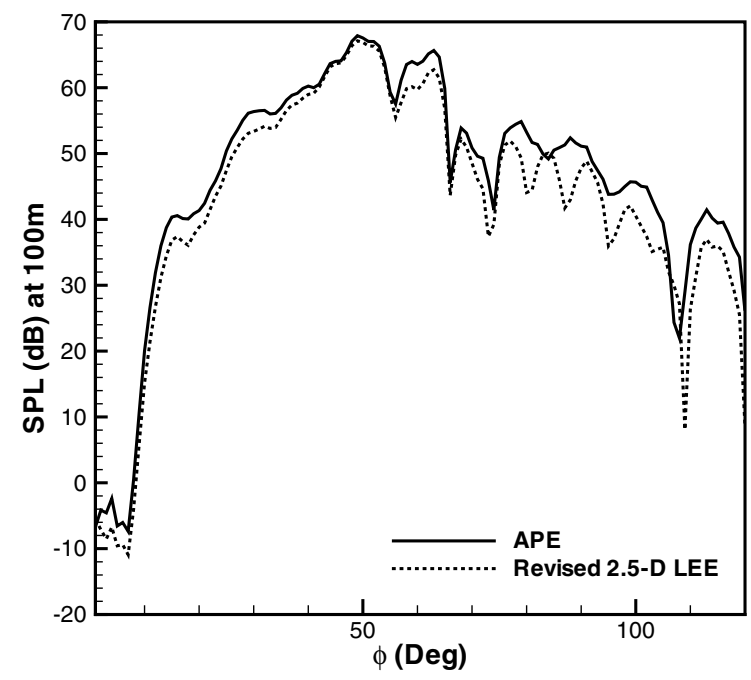

a) $n=1$

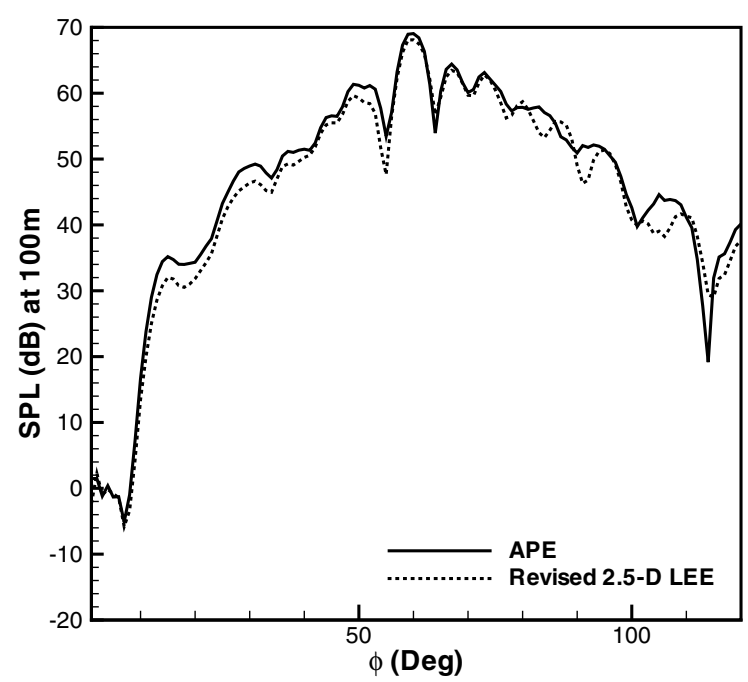

c) $n=3$ coordinate transformation, which represents a transformation from the physical to the computational coordinates. In this work, the AMR operations were conducted on the body-fitted multiblock meshes to increase grid resolution along with the sound propagation and to reduce the unnecessary blocks/grids to save computational costs. The procedure is illustrated in Fig. 10, where the APE model was used. The total number of grid points increases from 36,000 to 180,000 . The computation was executed and tested on a computer with a Pentium IV $3.0 \mathrm{GHz}$ CPU and 2 GB memory. Table 1 shows that the computation time of AMR is increased along with the increase of grid points. In the initial stage $(t<5)$, the computation time using AMR is around $100 \%$ faster than the computation time on the uniform mesh. After that, the computational efficiency of AMR gradually decreases. Finally, it reaches the same level of the computational efficiency on the uniform mesh, due to the extended span of the acoustic wave in the whole computational domain, where the AMR operations are not used anymore.

\section{Near-Field Propagation}

Figure 11 compares the near-field wave propagation computed by 2.5-D LEE [Eq. (3)] and APE [Eq. (12)], respectively. After $t=12$, the hydrodynamic instabilities developed in the shear layer are evident by using the 2.5 -D LEE model (see Fig. 11a). It was found that neither a filtering nor a damping technique can prevent the instability waves from overwhelming the desired acoustic solutions.

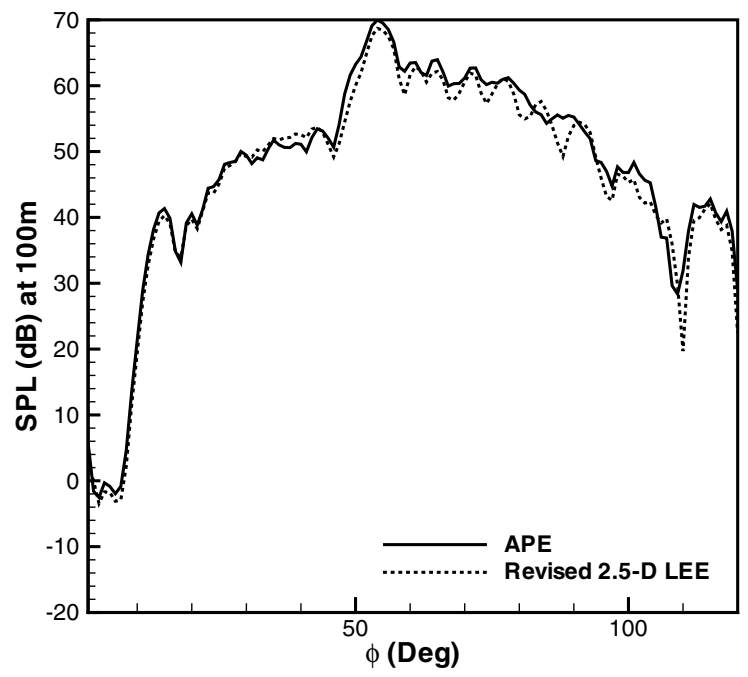

b) $n=2$

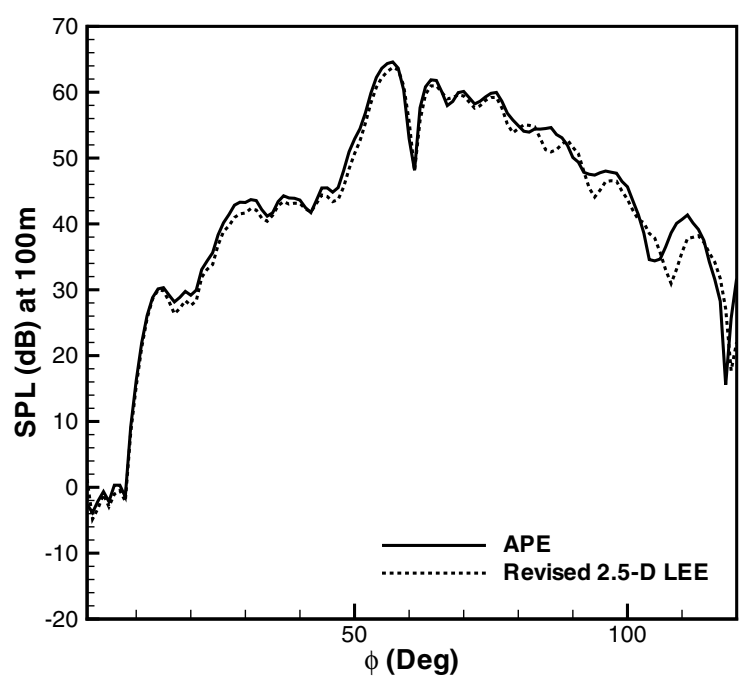

d) $n=4$

Fig. 13 Far-field directivity of engine exhaust duct radiation; $m=13, k=28.3$. 
By contrast, the development of instabilities in the shear layer can be avoided using either revised 2.5-D LEE or APE. Figure $11 \mathrm{~b}$ shows an example that was solved with the APE model. It can be seen that the numerical instabilities do not appear in the figure, even after longer sound wave propagation duration.

Figure 12 compares solutions from the revised 2.5-D LEE and the APE predictions. To present the pressure contours in the vicinity of the engine duct clearly, enlarged views are displayed in Figs. 12a and $12 \mathrm{c}$. SPL results obtained by two models are displayed in Figs. $12 \mathrm{~b}$ $\overline{\text { and }} 12 \mathrm{~d}$, respectively. Figure 12 shows that propagation patterns predicted by both methods agree well in most parts, and the key features, such as wave diffractions off the lip of the bypass duct and reflections off the surface of the afterbody of the engine exhaust, are similar. The SPL of the APE prediction is slightly higher at high and low angles than the revised 2.5-D LEE prediction. Several other spinning mode waves, that is $n=2-4$, were also solved using these two models and similar findings were reached.

\section{Far-Field Directivity}

The far-field directivities of the four spinning mode radiations were predicted through an integral solution of the FW-H equation based on the near-field solutions that were solved with either the revised 2.5-D LEE or the APE models. The predictions by both models are compared in Fig. 13. Results show that both patterns are similar. The main radiation angel and the level of the APE prediction match the LEE solutions well. The differences in the peak radiation level between the APE results and the LEE results are less than $0.5 \mathrm{~dB}$, whereas the peak radiation angles differ from each other by less than $1.4 \mathrm{deg}$. In other parts of the directivity, the patterns are also similar. The curve of the APE results, for the most part, is smoother than the curve of the LEE results. Another point to note is that the level of the APE results is generally higher than that of the revised 2.5-D LEE results. Here, the maximal difference appears in the case of $n=1$, where the difference at the high angles $(\phi>60 \mathrm{deg})$ is around $4.0 \mathrm{~dB}$. It is still an open problem to determine which model can produce more accurate results. To answer the question, a test case solving fully 3-D Navier-Stokes equations might be required.

\section{Summary}

This paper outlines two computational models for spinning modal sound propagation in, and radiation from, a duct with flow. The sound propagation inside the duct, refraction in the shear layer behind the lip of the duct, and propagation in the near field could be predicted by the 2.5-D LEE model, which, however, describes not only the acoustic mode but also the hydrodynamic mode. Numerical components associated with the hydrodynamic mode can be amplified exponentially in the shear flow to overwhelm the desired acoustic solution rapidly. To suppress the instabilities, several gradient terms were removed from LEE. An alternative APE model, which applies an acoustic filtering matrix on LEE to remove nonacoustic modes, was also used. Based on 2.5-D LEE, the APE model was extended to the cylindrical coordinates for axisymmetrical duct radiation problems considered in this work. Good agreement between the revised LEE and the APE models was demonstrated on a canonical case of sound radiation out of a semi-infinite duct with flow. The two models were then applied to a more general case of sound radiation out of a generic engine duct with flow. The body-fitted multiblock AMR method was applied for this case to solve the problem more efficiently. The computational efficiency varies along with the propagation of the acoustic waves. In the initial stage, the adaptively refined mesh represents a savings of up to $160 \%$ compared with a uniform mesh. After the acoustic waves span the whole computational domain, the efficiency of AMR is the same as that on a uniformly fine mesh. The results show that both models can predict the near-field sound propagation and the far-field sound directivity. The APE model, however, is more favorable for its suitability to arbitrary background flow.

\section{Acknowledgments}

Xun Huang and Zhaokai Ma were supported by scholarships from the School of Engineering Sciences, University of Southampton during this work. We also would like to acknowledge Roland Ewert of the German Aerospace Center and Anurag Agarwal of the Institute of Sound and Vibration Research for helpful discussions.

\section{References}

[1] Gabard, G., and Astley, R. J., "Theoretical Model for Sound Radiation from Annular Jet Pipes: Far- and Near-Field Solutions," Journal of Fluid Mechanics, Vol. 549, 2006, pp. 315-341. doi:10.1017/S0022112005008037

[2] Lidoine, S., Batard, H., Troyes, H., Delnevo, S., and Roger, M., "Acoustic Radiation Modelling of Aeroengine Intake Comparison Between Analytical and Numerical Methods," AIAA Paper 2001-2140, 2001.

[3] Eversman, W., and Okunbor, D., "Aft Fan Duct Acoustic Radiation," Journal of Sound and Vibration, Vol. 213, No. 2, 1998, pp. 235-257. doi:10.1006/jsvi.1997.1480

[4] Astley, R., Hamilton, J., Baker, N., and Kitchen, E., "Modelling Tone Propagation from Turbofan Inlets: The Effect of Extended Lip Liners," AIAA Paper 2002-2449, 2002.

[5] Tam, C. K. W., and Webb, J. C., "Dispersion-Relation-Preserving Finite Difference Schemes for Computational Acoustics," Journal of Computational Physics, Vol. 107, No. 2, 1993, pp. 262-281. doi:10.1006/jcph.1993.1142

[6] Hixon, R., "Prefactored Small-Stencil Compact Schemes," Journal of Computational Physics, Vol. 165, No. 2, 2000, pp. 522-541. doi:10.1006/jcph.2000.6631

[7] Richards, S. K., Chen, X. X., Huang, X., and Zhang, X., "Computation of Fan Noise Radiation Through an Engine Exhaust Geometry with Flow," International Journal of Aeroacoustics, Vol. 6, No. 3, 2007, pp. 223-241. doi: $10.1260 / 147547207782419534$

[8] Leneveu, R., Schiltz, B., Manera, J., and Caro, S., "Parallel DGM Scheme for LEE Applied to Exhaust and Bypass Problems," AIAA Paper 2007-3510, 2007.

[9] Zhang, X., Chen, X. X., Morfey, C. L., and Nelson, P. A., "Computation of Spinning Modal Radiation from an Unflanged Duct," AIAA Journal, Vol. 42, No. 9, 2004, pp. 1795-1801.

[10] Zhang, X., Chen, X. X., and Morfey, C. L., "Acoustic Radiation from a Semi-infinite Duct with a Subsonic Jet," International Journal of Aeroacoustics, Vol. 4, Nos. 1-2, 2005, pp. 169-184. doi:10.1260/1475472053730075

[11] Richards, S. K., Zhang, X., Chen, X. X., and Nelson, P. A., "Evaluation of Non-Reflecting Boundary Conditions for Duct Acoustic Computation," Journal of Sound and Vibration, Vol. 270, No. 3, 2004, pp. 539-557. doi:10.1016/j.jsv.2003.09.042

[12] Huang, X., Zhang, X., and Richards, S. K., "Adaptive Mesh Refinement Computation of Acoustic Radiation from an Engine Intake," Aerospace Science and Technology (in press), 2008. doi:10.1016/j.ast.2007.09.004.

[13] Agarwal, A., Morris, P. J., and Mani, R., "Calculation of Sound Propagation in Nonuniform Flows: Suppression of Instability Waves," AIAA Journal, Vol. 42, No. 1, 2004, pp. 80-88.

[14] Casalino, D., and Genito, M., "Turbofan Aft Noise Predictions Based on Lilley's Wave Model," AIAA Journal, Vol. 46, No. 1, 2008, pp. 84 93.

doi:10.2514/1.32046

[15] Munt, R. M., "Interaction of Sound with a Subsonic Jet Issuing from a Semi-infinite Cylindrical Pipe," Journal of Fluid Mechanics, Vol. 83, 1977, pp. 609-640. doi:10.1017/S0022112077001384

[16] Bogey, C., Bailly, C., and Juvé, D., "Computation of Flow Noise Using Source Terms in Linearized Euler's Equations," AIAA Journal, Vol. 40, No. 2, 2002, pp. 235-243.

[17] Ewert, R., and Schröder, W., "Acoustic Perturbation Equations Based on Flow Decomposition via Source Filtering," Journal of Computational Physics, Vol. 188, No. 2, 2003, pp. 365-398. doi:10.1016/S0021-9991(03)00168-2

[18] Ewert, R., and Schröder, W., "On the Simulation of Trailing Edge Noise with a Hybrid LES/APE Method," Journal of Sound and Vibration, Vol. 270, No. 3, 2004, pp. 509-524.

doi:10.1016/j.jsv.2003.09.047

[19] Pierce, A. D., "Wave Equation for Sound in Fluids with Unsteady 
Inhomogeneous Flow," Journal of the Acoustical Society of America, Vol. 87, No. 6, 1990, pp. 2292-2299. doi:10.1121/1.399073

[20] Berger, M. J., and Oliger, J., "Adaptive Mesh Refinement for Hyperbolic Partial Differential Equations,” Journal of Computational Physics, Vol. 53, No. 3, 1984, pp. 484-512. doi:10.1016/0021-9991(84)90073-1

[21] Sachdev, J. S., Groth, C. P. T., and Gottlieb, J. J., "Parallel SolutionAdaptive Scheme for Predicting Multi-Phase Core Flows in Solid Propellant Rocket Motors," International Journal of Computational Fluid Dynamics, Vol. 19, No. 2, 2005, pp. 159-177. doi:10.1080/10618560410001729135

[22] MacNeice, P., Olson, K. M., Mobarry, C., deFainchtein, R., and Packer, C., "PARAMESH: A Parallel Adaptive Mesh Refinement Community Toolkit," Computer Physics Communications, Vol. 126, No. 3, 2000, pp. 330-354. doi:10.1016/S0010-4655(99)00501-9

[23] Farassat, F., and Succi, G. P., Prediction of Helicopter Rotor Discrete Frequency Noise, American Helicopter Society, Annual Forum, 38th, American Helicopter Society, Washington, D.C., 1982, pp. 497-507.

[24] Brentner, K. S., "Numerical Algorithms for Acoustic Integrals with Examples for Rotor Noise Prediction," AIAA Journal, Vol. 35, No. 4, 1998, pp. 625-630.

[25] Ashcroft, G., and Zhang, X., "Optimized Prefactored Compact Schemes," Journal of Computational Physics, Vol. 190, No. 2, 2003, pp. 459-477.

doi:10.1016/S0021-9991(03)00293-6

[26] Hu, F. Q., Hussaini, M. Y., and Manthey, J., "Low-Dissipation and Low-Dispersion Runge-Kutta Schemes for Computational Acoustics," Journal of Computational Physics, Vol. 124, No. 1, 1996, pp. 177-191. doi:10.1006/jcph.1996.0052

[27] Homicz, G. F., and Lordi, J. A., "Note on the Radiative Directivity Patterns of Duct Acoustic Modes," Journal of Sound and Vibration, Vol. 41, No. 3, 1975, pp. 283-290. doi:10.1016/S0022-460X(75)80175-1

[28] Huang, X., and Zhang, X., "Adaptive Mesh Refinement for Computational Aeroacoustics," AIAA Paper 2005-2873, 2005.

[29] Driscoll, T. A., and Trefethen, L. N., "Pseudospectra for the Wave Equation with an Absorbing Boundary," Journal of Computational and Applied Mathematics, Vol. 69, No. 1, 1996, pp. 125-142. doi:10.1016/0377-0427(95)00021-6

[30] Mittal, R., and Iaccarino, G., "Immersed Boundary Methods," Annual Review of Fluid Mechanics, Vol. 37, Jan. 2005, pp. 239-361. doi:10.1146/annurev.fluid.37.061903.175743

[31] Berger, M. J., and Jameson, A., "Automatic Adaptive Grid Refinement for the Euler Equations," AIAA Paper 1985-1633, 1985.

C. Bailly Associate Editor 